\title{
LA DIFERENCIA ENTRE MOSTRAR Y DECIR Y EL PROBLEMA DEL SIGNIFICADO
}

\section{The Difference between Showing and Saying and the Problem of Meaning}

\author{
Carlos E. CaOrsi ${ }^{a}$ \\ cecaorsi@hotmail.com \\ ${ }^{a}$ Instituto de Filosofía, FHCE/UDELAR, Montevideo, Uruguay
}

\begin{abstract}
Resumen
En este artículo me interesa considerar la conclusión de Moretti, según la cual hay una disolución del problema de la unidad de la proposición en términos de la noción de interpretación, que parece obviar su solución tradicional basada en relaciones referenciales, pero que, sin embargo, o bien requiere ese tipo de fundamentación, o bien hace lugar a planteamientos trascendentales que la exceden. Coincido mayormente con esta conclusión, pero creo del caso desarrollar algunas consideraciones que me apartan en cierta medida de algunas de las suyas. En particular, me centraré en la distinción ente decir y mostrar, y en la interesante extensión por parte de Moretti de esta distinción, formulada habitualmente para las oraciones, a la situaciones de enunciación. Sostendré, contra lo que él afirma, que la imposibilidad de decir lo que la enunciación muestra es menos radical que la imposibilidad de decir lo que muestra una oración.
\end{abstract}

Palabras clave: Unidad de la proposición; Mostrar/decir; Enunciación;Triangulación.

\begin{abstract}
In this paper I am interested in considering Moretti's conclusion, according to which there is a dissolution of the problem of the unity of the proposition in terms of the notion of interpretation, which seems to obviate the traditional solution based on referential relations, but which, nevertheless, either requires that kind of foundation or makes room for transcendental approaches that go beyond it. I mostly agree with this conclusion but I think it is appropriate to develop some considerations that separate me to some extent from some of his. In particular, I will focus on the distinction between saying and showing, and on Moretti's interesting extension of this distinction, usually formulated for sentences, to situations of enunciation. Against his position, I will maintain that the impossibility of saying what the enunciation shows is less radical than the impossibility of saying what a sentence shows.
\end{abstract}

Key words: The Unit of the Proposition; Showing/saying; Enunciation; Triangulation. 
Moretti comienza su artículo "La unidad proposicional" con la siguiente afirmación:

Se sostendrá que hay una disolución del problema [de la unidad de la proposición] en términos de la noción de interpretación, que parece obviar su solución tradicional basada en relaciones referenciales. Pero que, sin embargo, el marco general de esa disolución -las condiciones de una interpretación en general-o bien requiere ese tipo de fundamentación, o bien hace lugar a planteamientos trascendentales que la exceden. (Moretti 2014, p. 2)

En lo que sigue me propongo considerar esta conclusión, con la que estoy básicamente de acuerdo, a los efectos de realizar algunas puntualizaciones que me parece del caso enunciar. De hecho, voy a ampliar mis consideraciones al problema general del significado, del cual el problema de la unidad de la proposición es una parte - aunque se trata de una parte vitalmente central desde el momento en que la adquisición del significado comienza con proferencias de oraciones. Así, la construcción de una teoría del significado supone, como paso fundamental, el modo en que el significado de las partes de la oración contribuye al significado de la oración completa, y éste es básicamente el problema de la unidad de la proposición.

El modo en que Moretti desarrolla su argumentación se basa en dos distinciones; a saber, una distinción de los enfoques con que suele abordarse el problema, y una distinción relativa a lo que una oración hace, o mejor dicho, lo que con una oración se hace, aun cuando por lo general el que lo hace no sospeche que lo está haciendo. La primera distinción, la distinción entre enfoques, consiste en la siguiente: (I) un enfoque LM que pretende dar cuenta de la unidad de la proposición apelando a los referentes de los componentes de la oración; este enfoque "da un lugar privilegiado a los nexos entre el lenguaje, el pensamiento y la realidad" (Moretti 2014, p. 3) ${ }^{1}$; (II) un enfoque IL, que enfatiza el problema "de la posibilidad de la comprensión y enfoca el lenguaje como un sistema de interpretaciones" (Moretti 2014, p. 3). La segunda distinción es la

\footnotetext{
${ }^{1}$ Un punto central de la cuestión, que por cierto Moretti señala, es que, por lo menos en el caso de Davidson, la comprensión-interpretación da un lugar privilegiado a los nexos entre intérpretes, lenguaje y mundo, los cuales juegan un papel central en el proceso de la triangulación.
} 
que se establece entre "decir" y mostrar". Parece ser el caso que ciertas expresiones nos permiten entender lo que no podemos decir con ellas. La teoría figurativa del lenguaje desarrollada en el Tractatus LogicoPhilosophicus de Wittgenstein abunda en esta distinción y la hace parte fundamental de su teoría. ${ }^{2}$ Así, por ejemplo, dice Wittgenstein (1921):

(2.17) Lo que la figura ha de tener en común con la realidad para poder figurarla a su modo y manera -correcta o falsamente- es su forma de figuración.

(2.72) Pero la figura no puede figurar su forma de figuración, la ostenta.

Moretti, por su parte, utiliza el siguiente ejemplo: “Jantipa pasea', dice que Jantipa pasea, pero muestra la instanciación del universal Pasea, o la aplicación del predicado pasea (Moretti 2014, p. 7). Y señala que al comprender lo dicho se entra en relación con algo mostrado por la enunciación. Y agrega que no podría haber comprensión de lo dicho sin algún modo de experiencia de lo mostrado.

Este punto es sin duda fundamental para entender el fenómeno del lenguaje, y desentenderse del mismo conduce por caminos poco auspiciosos. Me interesa sobre todo el fenómeno de la comprensión y su relación con la experiencia de lo mostrado, destacado por Moretti en esta caracterización. Supongamos que un niño, aprende a proferir "Gavagai" como una de sus primeras palabras. El niño, en una primera instancia, simplemente ha sido condicionado a asociar el sonido "Gavagai" con algo que se le muestra en esa ocasión. Diríamos que lo que se le muestra es la presencia de un conejo, pero el niño no puede decirlo. Lo que el niño puede decir es "Gavagai". Un traductor radical con su propio lenguaje a disposición podrá decir 'conejo', o 'parte no separada de conejo' o cosas por el estilo. ${ }^{3}$ Pero lo que se le presenta al niño en esa ocasión, aquello

\footnotetext{
${ }^{2}$ Moretti atribuye la prioridad de esta distinción a Frege y dada su elocuente respuesta a la pregunta que le hiciera al respecto no tengo razones para dudar de ello. Pero como el tiempo apremia y no he podido disponer del suficiente para rastrear esos pasajes en Frege, me limito a mencionar los que pueden ubicarse más fácilmente, debidos a Wittgenstein. Creo que Moretti sabrá disculpar esta negligencia, debida más a la tiranía del tiempo que a la pereza.

${ }^{3}$ Soy consciente de que no estoy planteando las cosas con la debida precisión ya que he obviado la diferencia entre "Conejo" y 'conejo', es decir entre el uso de la oración observacional y el uso del término. Y que las diferencias empiezan, según Quine, en este ultimo caso y no en el que comencé presentando. Como él señala, las oraciones observaciones son "moneda universal"; las diferencias aludidas en el ejemplo entre distintas traducciones devienen con posterioridad con la adquisición
} 
de lo que tiene experiencia, es lo que recurrentemente se le presenta cada vez que su maestro profiere "Gavagai" y lo que se le presenta cuando es premiado por su proferir "Gavagai". El resto es historia conocida, adquisición del aparato de individuación, etc. y a partir de allí los alternativos quineanos de 'conejo', 'parte no separada de conejo', 'conejofanía' y otros por el estilo. Y la pregunta quineana: ¿'Conejo' en qué sentido de 'conejo'? y el supuestamente necesario regreso al lenguaje de fondo, que funcionará como un sistema de coordenadas. Para llegar finalmente a la conclusión: no tiene sentido decir lo que significa una palabra sino relativamente a un sistema de coordenadas, o lenguaje de fondo. Y el lenguaje de fondo por excelencia será el lenguaje materno; pero no, a mi modo de ver, como podría pensarse, como resultado de una opción pragmática por lo más familiar para evitar un regreso al infinito, sino porque no podría ser de otro modo. Porque es en ese, nuestro primer lenguaje, que el significado se muestra, no se dice. El significado se asienta sobre lo que se muestra, y olvidar este hecho es lo que conduce a las preguntas del tipo ¿qué quiere decir con eso? Y la única respuesta no inocente parece ser: precisamente eso.

No pretendo con esto ignorar la complejidad resultante de la conformación de un lenguaje y la necesaria estructura sintáctica que esto supone, así como las distintas categorías semánticas y la correspondiente forma lógica. A eso aluden los ejemplos de Wittgenstein y Moretti que he referido anteriormente. Como Moretti señala "Jantipa pasea", muestra la instanciación del universal Pasea, o las aplicaciones del predicado 'pasea', dependiendo por cierto de nuestra preferencia entre Platón y Aristóteles. Pero el modo en que Jantipa se ha presentado en las instancias de adquisición de 'Jantipa' (supuesto que la hayamos adquirido por familiaridad, para usar una expresión russelliana) y el modo en que se ha presentado 'pasea' (bajo la misma suposición) en las circunstancias de su adquisición, son diferentes. $\mathrm{Y}$ esta diferencia es

del aparato de individuación del lenguaje. El problema de la referencia se presenta, por así decirlo, una vez adquirido el referido aparato. Ello no obsta, sin embargo, para que Quine haga observaciones como la siguiente: "Pero la referencia está afuera en el mundo antes y después, en el vértice "ello" del triángulo. El niño simplemente tiene que aprender más acerca de ella. Mientras tanto el rango de las entradas neurales que promueven el asentimiento del niño a la oración observacional "Pelota" pueden permanecer incambiadas." (Quine 2000, pp. 1-6, las cursivas me pertenecen). Tal vez al establecer esta diferencia entre la referencia antes y después, esté considerando la diferencia entre lo que en primera instancia se muestra y lo que después es ajustado por el lenguaje. Pero la persistencia de Quine en las explicaciones neurofisiológicas propias de su postura naturalista hacen difícil suponer que lo haga. Y al no hacerlo la afirmación no deja de presentar el aire paradojal que sin duda ostenta. 
la que permite que "Jantipa pasea" muestre lo que muestra. Al asumir que el modo en que Jantipa se presenta es distinto del modo en que lo hace 'pasea', estoy asignando prioridad al mundo sobre el lenguaje, pero estoy convencido de que esa prioridad es lo que hace posible el lenguaje. Si el mundo, como señala Austin, no exhibiera similitudes y diferencias, el lenguaje no sería posible. El modo en que algunos sostienen que el lenguaje "rebana" el mundo se parece menos a cortar una torta que a romper un cristal.

Pero dejemos ya estas disquisiciones, que no estoy totalmente seguro de que Moretti comparta, y volvamos a otra aplicación que él hace de la distinción mostrar/decir, que me resulta sumamente iluminadora. El modo más habitual de establecer la distinción es aplicarla a oraciones, y sobre ello ya hemos realizado algunos comentarios. Pero, en su artículo, Moretti aplica esta misma distinción a las situaciones de enunciación. Así como una oración muestra lo que no dice, lo mismo, señala Moretti, sucede con la enunciación. A propósito afirma: "Se advierte así que, [...], lo que una enunciación muestra es el ser hablante-intérprete, del hablante-intérprete. Y en una enunciación asertiva (una aserción) se muestra, en general, lo que podemos llamar la estructura intérprete-cosas-relaciones" (Moretti 2014, p. 8). De acuerdo con esto, lo que la enunciación muestra, independientemente de cuál sea su contenido (lo que dice), es la estructura intérprete-cosas-relaciones. Parece importante señalar que para entender lo que un enunciado $p$ dice debo:

(i) interpretarlo, y para interpretarlo debo

(ii) presuponer la estructura intérprete-cosas-relaciones. Pero interpretarlo presupone

(iii) la función mostrativa de $p$ y tener experiencia de lo mostrado por $p$.

Resumamos lo dicho hasta ahora. La distinción se aplica a dos cosas diferentes:

(i) a las oraciones, que muestran, para usar una expresión de Wittgenstein, su modo de figuración;

(ii) a las enunciaciones, que muestran la estructura intérpretescosas-relaciones.

Para el caso de (ii), podríamos decir que la capacidad de interpretar lo que un hablante dice presupone esta estructura. De acuerdo 
con esto, lo que una teoría lógico-semántica se propone es representar los rasgos constitutivos de esta estructura que los actos asertivos muestran. Pero, como es sabido, las teorías lógico-semánticas se expiden acerca del lenguaje en el que ellas mismas se desarrollan y con ello son, al decir de Moretti, "presumiblemente incompletables". En virtud de esta incompletud lo que las teorías parecen mostrar es que la estructura del lenguaje o del mundo, presupuesta como totalidad, no es cognoscitivamente accesible, no es objeto de conocimiento, sino presuposición de la práctica de construir teorías. Con esto llegamos a la tesis que Moretti formula del siguiente modo: "La estructura formada por la relación entre las comunidades de hablantes, el sistema lingüístico y la trama de las cosas, donde ninguna de estas estructuras es más fundamental que las otras, es la presuposición fundamental de los actos interpretativos." (Moretti 2014, p. 9)

Ahora bien ¿qué podemos decir de esta estructura presupuesta? De acuerdo con Moretti, podemos concebir dicha estructura de dos modos:

(i) como parte de lo que puede decirse;

(ii) como lo que posibilita todo decir.

En el caso (i), la estructura es objetivada (como sucede en las perspectivas naturalistas). Como resultado de esta objetivación, la estructura intérprete-cosas-relaciones se piensa como un acontecimiento del mundo del cual es posible decir algo. Y esto a su vez puede pensarse de dos modos:

(i) desde un enfoque ML, en el cual podemos referirnos a la estructura y sus partes constitutivas;

(ii) a la manera de Davidson, desde un enfoque IL reformulado mediante propiedades y relaciones, como las de referencia, verdad, consecuencia lógica, percepción, intencionalidad, etc. pensadas sustantivamente y no como meros instrumentos (como según Moretti hace Davidson) para obtener la interpretación; es decir, insertando los conceptos (nombre, oración, aserción, significado, etc.) y tesis que exponen el problema de la unidad en una trama explícita que va más allá de una teoría "explícitamente semántica".

Respecto de la perspectiva (i), dirá Moretti: "Pero siendo [dicha estructura] lo presupuesto por el habla no presupone el habla (aunque esté "simultáneamente" dado con ella). De este modo, el vínculo lenguaje/ mundo es un objeto y presupone el habla y no presupone el habla y no es un objeto" (Moretti 2014, p. 10). En lo que respecta al caso (ii), afirma: "Al pensar lo que hace posible que haya objetos para nosotros, 
pensamos en un objeto (por complejo que sea). Entonces no pensamos lo que queríamos" (Moretti 2014, p. 10). Y más adelante,

Si el vínculo intérprete/mundo no se requiere pensar como un acontecimiento del mundo, esto es como algo de lo que puede decirse algo, sino como lo que hace posible decir, o como la posibilidad de decir sobre el mundo, tampoco parece que pueda decirse nada (ni siquiera nombrarlo) sin caer en contradicción pragmática. Esto se expone, a veces, como la constatación de la inefabilidad de la semántica, o cómo el fenómeno de la intraspasibilidad para el pensamiento. Como un "límite" del pensar. (Moretti 2014, p. 10)

Y pregunta a continuación: ¿qué indica esta inescapabilidad? Y sus respuestas son: para algunos es, simplemente, otra descripción del hecho de haber fijado un lenguaje de fondo (que podría sustituirse más o menos ad necessitatem); para otros señala hacia algo que está "fuera" de lo que llamamos lenguaje de fondo y mundo y que, sin embargo los hace posibles. Para pasar a concluir: "En ambos casos parece infructuoso el intento de pasar desde la equívoca tesis de que hay algo presupuesto, aún si es entendida "metafóricamente", hasta alguna determinación específica necesaria de sus características" (Moretti 2014, p. 11).

En lo que sigue me interesa detenerme en esta última conclusión según la cual parece no ser posible pasar de la tesis de que hay algo presupuesto a alguna determinación específica de sus características. Para ello será preciso detenerse en los enfoques LM e IL, y en la distinción decir/mostrar.

Comencemos por esta última distinción. La pregunta que se nos plantea es: ¿nunca puede decirse lo que se muestra? Si considero el caso de lo que una oración muestra, creo que la respuesta es afirmativa. Todo decir lo que una expresión significa es una ilusión fundada en una aceptación acrítica de un lenguaje de fondo. Para recurrir nuevamente a Russell ${ }^{4}$, toda palabra se aprende o bien por familiaridad (acquaintance), o bien por descripción. En el caso de la familiaridad, el significado se muestra y no hay modo en que se diga; recordemos las observaciones hechas más arriba respecto del niño que aprende "Gavagai" como una de

\footnotetext{
${ }^{4}$ Aunque sería más adecuado para nuestros intereses adoptar en este caso una lectura externista de la distinción russelliana.
} 
sus primeras palabras. Si fuera posible preguntarle al niño qué quiere decir con "Gavagai", este sólo podría responder 'esto' (acompañado de un gesto de ostensión), o simplemente "Gavagai". En el caso del aprendizaje por descripción, ya se presupone la adquisición de una porción significativa del lenguaje. Pero aun así, los predicados utilizados para dar el significado buscado deben haberse adquirido por familiaridad (mostración) o por descripción en base a otros predicados que hemos de haber adquirido por familiaridad. En todo caso y por mediatizado que sea el retorno, el punto final del mismo es lo que se muestra.

Pero ¿cuál sería la respuesta respecto de lo que muestra un acto de enunciación? En este caso, lo que se muestra no es el significado, sino una cierta estructura que, siguiendo a Moretti, llamaremos, 'intérpretescosas-relaciones'. Claro que para poder describir esta estructura es necesario un lenguaje y con ello todo lo que esto supone, pero tengo la sospecha de que la imposibilidad de decir es, en este caso, menos radical de la que se presenta en el primer caso.

A propósito de esto me resulta algo enigmática la afirmación de Moretti, según la cual "es posible que N (una teoría) sea verdadera y diga que el cosmos se las arregló la mayor parte de su existencia sin lenguaje $\mathrm{y}$ sin hablantes, pero sin lenguaje y sin intérpretes $\mathrm{N}$ no dice nada, $\mathrm{y}$ por ende, no puede ser verdadera" (Moretti 2014, p. 11). Y me resulta enigmática, en el siguiente sentido. Podría entenderla como afirmando que es posible que $\mathrm{N}$ (una teoría) sea verdadera y diga que el cosmos se las arregló la mayor parte de su existencia sin lenguaje y sin hablantes. Esta parte del enunciado tomada aisladamente me parece verdadera y no problemática. Por otra parte, la siguiente afirmación, según la cual sin lenguaje y sin intérpretes una teoría $\mathrm{N}$ no dice nada, y por ende, no puede ser verdadera, me parece también verdadera y no problemática, si la entiendo como un modo un poco idiosincrático de decir que sin lenguaje ni intérpretes no puede haber teorías, dado que una teoría supone un lenguaje y un lenguaje supone hablantes-intérpretes. Sin embargo el 'pero' que vincula ambas afirmaciones hace dudoso que este modo de entenderlas sea correcto. ¿Quiere decir que no puede haber teorías que traten del mundo antes de la aparición del lenguaje? Por cierto, no me atrevo a decir que este sea el caso. ¿Quiere decir que no hay mundo si no hay lenguajes? Parece difícil atribuirle a Moretti la creencia de que en principio fue el verbo. Parece más verosímil suponer que lo que está diciendo es que conceptos como los de mundo, objeto, etc., sólo se dan una vez que disponemos de un lenguaje con todo lo que esto supone. Y si este es el caso, que dado el modo en que se da la relación lenguaje-mundo, la idiosincrasia del lenguaje haría imposible decir cómo sería el mundo si 
uno se situara en un punto exterior al lenguaje —en el cual es imposible situarse. Me inclino a pensar que este es el caso, lo cual coincide con la siguiente afirmación de Moretti: "Mundo y lenguaje aparecen "a la vez": las leyes lógico-semánticas ni sólo son constitutivas del lenguaje ni se derivan de leyes fundamentales del mundo" (Moretti 2014, p. 12). Lo cual aparece más desarrollado en la conclusión donde afirma:

No hay nociones generales de objeto o relación que puedan integrar un corpus teórico fundacional; esas palabras (cuya intelección, i.e., su carácter de palabras, presupone la estructura intérpretes-mundo) no aluden a conceptos teóricos sino que son indicaciones o gestos para suspender la mirada que normalmente se dirige hacia (o es dirigida por) lo que se dice, dando lugar a la mirada hacia el decir mismo, que conduce a "mirar" que hay lenguaje y mundo (en lugar de nada). Este mirar es teóricamente indecible porque intentar decirlo (teóricamente) sería intentar hacer presente, sólo diciéndolo, lo que se caracteriza como lo que no presupone el decir pero es presupuesto por todo decir. (Moretti 2014, p. 10)

\section{4}

Visto esto quisiera volver a la diferencia mostrar/decir y a la sospecha, esbozada en 3 , de que la imposibilidad de decir lo que la enunciación muestra es menos radical que la imposibilidad de decir lo que muestra una oración. Para ello me interesa hacer algunas consideraciones acerca de la propuesta de Davidson que Moretti incluye dentro de la perspectiva IL.

Si bien no hay cambios radicales en el desarrollo de las propuestas de Davidson a lo largo de su obra, creo que es posible señalar algunas diferencias entre un Davidson temprano, que enfatiza la construcción de teorías $\mathrm{T}$ como teorías del significado, algo que por cierto nunca abandona, y un Davidson más tardío, que hace un especial hincapié en el proceso de triangulación. No se trata por cierto de un cambio teórico sustancial, ya que el modelo de interpretación basado en teorías T va del principio al final y sucede lo mismo con el proceso de interpretación. Yo hablaría en todo caso de una diferencia fundamentalmente de énfasis entre un período y otro.

Respecto del Davidson temprano, podemos incluirlo sin duda en la perspectiva IL, como lo hace Moretti, pero no estoy tan seguro de que podamos decir que llega a una disolución del problema de la unidad proposicional en el modo en que Moretti lo plantea, aunque hay algunas 
observaciones que podrían avalar dicha afirmación. Sin embargo, yo me inclinaría por sostener que el método de Tarski le permitió dar una explicación parcial del problema de la predicación. Veamos con cierto detenimiento cómo se da el planteo de la cuestión en las conferencias Hermes dictadas en la Universidad de Perugia en mayo de $2001 .^{5}$

En este texto observa que una revisión de la historia del problema de la predicación revela cuatro condiciones que debe cumplir una solución satisfactoria de problema de la predicación. Las mismas son:

(i) una explicación satisfactoria depende de relacionar la predicación con la verdad de las oraciones; ${ }^{6}$

(ii) proveer una semántica para los predicados, asociándolos con objetos, como universales, propiedades o conjuntos, no soluciona el problema porque siempre conduce a un regreso infinito; ${ }^{7}$

(iii) es esencial separar la observación obvia de que los predicados introducen la generalidad de la oraciones, del pensamiento de que los predicados deben al mismo tiempo introducir universales $\mathrm{u}$ otras entidades abstractas en el tema de las oraciones; ${ }^{8}$

(iv) el problema de la predicación emerge sólo en conjunción con una concepción clara de la forma lógica de las oraciones hasta que no tengamos esta concepción estaremos inseguros respecto de qué cuenta como un término singular o un predicado. $^{9}$

Y la solución propuesta por Davidson cumple con estos requisitos: toma de Frege la relación exigida en (i) y la forma lógica exigida en (iv). Pero la propuesta de Frege no le permite evadir los puntos (ii) y (iii), en parte porque atribuye una referencia a las oraciones y en parte porque lo hace también respecto de los predicados. Las teorías de Tarski le permiten conservar los aportes de Frege sin la necesidad de asumir lo que (ii) y (iii) plantean que no debemos hacer. ${ }^{10}$ Las oraciones y los

${ }^{5}$ Incorporadas en Davidson (2005).

${ }^{6}$ Véase la página 141.

${ }^{7}$ Véase la página 143.

${ }^{8}$ Véase la página 145.

${ }^{9}$ Es posible que Moretti al hablar de la disolución del problema se esté refiriendo a lo que podría seguirse de esta condición, en el entendido de que una vez determinada la forma lógica "adecuada", la que adoptan las teorías T, el problema no se plantea. En este caso nuestra discrepancia por el uso del término "disolución” no tendría lugar.

${ }^{10}$ A propósito sostiene Davidson: "Hasta ahora, el método de Tarski no ha sido distinguido del de Frege excepto en que no asocia entidades que expresen generalidad 
predicados no denotan, sino que las primeras son verdaderas o falsas y los segundos son verdaderos de o falsos de. De este modo, una teoría T entendida como una teoría del significado da una solución al problema de la predicación. Sin embargo, parece que se plantea para esta solución algo similar a lo que sucede con la definición de verdad de Tarski. Una de las objeciones que se le han realizado, y que por cierto resulta verdadera, es que no define la verdad a secas, sino la verdad para un determinado lenguaje L. Y lo mismo parece resultar de la explicación de la predicación mediante el método T de Davidson. En Truth and Predication Davidson pregunta: ¿el método de Tarski para definir la verdad, modificado del modo en que he sugerido, soluciona el problema de la predicación? Y su respuesta es: “... es verdad que no surge ninguna explicación general de la predicación. Lo que surge es un método para especificar el rol de cada y cualquier predicado de un lenguaje específico" (Davidson 2005, p. 161, las cursivas me pertenecen).

Es en este sentido que sostengo que en Davidson lo que se da es una explicación parcial de la predicación para un lenguaje L y no una disolución del mismo. Tal vez la afirmación de que provee una disolución del problema pueda resultar del punto (iv), donde hace depender el problema de la especificación de la forma lógica. En ese caso podría decirse, si la forma lógica es la adecuada, el problema no se plantea.

Ahora quiero pasar a considerar el otro punto de la conclusión de Moretti, respecto de que esta disolución del problema presupone cuestiones que son características del enfoque LM. Creo que la conclusión es correcta, pero creo que esta suposición es hecha explícita por Davidson y de manera más clara en lo que llamamos el Davidson tardío. Después de desarrollar su solución del problema de la unidad de la proposición, Davidson sostiene que "la historia no está completa sin un examen de cómo es posible decir si un hablante, o grupo de hablantes, está usando un lenguaje definido por el método descrito" (Davidson 2005, p. 161). Y lo que se necesita para completarla es lo que Moretti ha denominado la 'estructura intérpretes-cosas-relaciones'. ${ }^{11}$

con predicados o algunas entidades cualesquiera con las oraciones. El foco sobre el papel de las variables o el espacio que ocupan es análogo al de Frege y fue inspirado por él. La innovación esencial de Tarski es hacer un uso ingenioso de la idea de que los predicados son verdaderos de las entidades que son nombradas por las constantes que ocupan sus espacios o de las que se cuantifica por medio de las variables que aparecen en el mismo espacio y están ligadas por cuantificadores" (Davidson 2005, p. 159).

${ }^{11}$ Las afirmaciones siguientes de Davidson hacen bastante explícito lo que falta: "De todas formas, frecuentemente podemos decir cuando alguien está haciendo una aserción honesta, o más en general, cuando un hablante esta profiriendo una oración que él o ella sostiene que es verdadera. Si este no fuera el caso, no podríamos nunca 
Por lo que acabamos de señalar, y por otras consideraciones que paso a desarrollar, resulta más difícil ubicar al Davidson tardío en alguna de las dos perspectivas propuestas, LM o IL.

Según señala Føllesdall (1999), se produce un cambio en la propuesta de Davidson a partir de una caminata que realizaron juntos en las colinas cerca de Biel, Suiza, en la cual discutieron el carácter indispensable de la percepción para la interpretación. Este reconocimiento del lugar de la percepción en la interpretación comienza a hacerse evidente en la publicación de 1982 de "Rational Animals", donde aparece mencionado por primera vez en forma escrita el problema de la triangulación. Es cierto que lo más cercano a un argumento sobre la triangulación lo encontramos recién en "Second Person" de $1992^{12}$, pero el tema comienza a dominar gran parte de los artículos de Davidson a partir de $1982 .{ }^{13}$ Este desarrollo de algún modo confirma la afirmación de Moretti respecto de que la respuesta al problema desde la perspectiva IL presupone la estructura intérpretes-cosas-relaciones. Porque, en efecto, podríamos entender el proceso de triangulación como una descripción de lo que el acto de enunciación muestra. Sólo que en este caso parece que Davidson no sólo presupone esa estructura sino que se propone decir algo de ella, para decirlo en palabras de Moretti, decir algo de lo que se muestra aun cuando ese decir lo presuponga.

Uno de los lugares en el que podríamos ver a Davidson como no ubicable exclusivamente en LM ni en IL, sino tal vez con un pie en cada una de ellas, es en las consideraciones desarrolladas en "Method and Metaphysics"14 de 1993 —las cuales me recuerdan en parte o tienen

\footnotetext{
llegar a entender un lenguaje. Patrones rudimentarios de oraciones sostenidas como siendo verdaderas pueden emplearse para identificar las constantes lógicas, conjunción, negación, y el aparato de la cuantificación. Algunos nombres son aprendidos por ostensión directa y como si estuvieran contenidos en oraciones: lo que puede decirse sin equívoco es justamente un nombre, entendido como una oración corta ("Este es Peter", "Este es Paul"). Los nombres aprendidos de este modo tienen una referencia garantizada. Los nombres aprendidos menos directamente pueden ser tratados como descripciones definidas. Esto deja de lado a los predicados. Como con los nombres, algunos predicados no estructurados pueden ser aprendidos por ostensión: de nuevo lo que es proferido pueden ser palabras solas, tratadas como oraciones ("Esto es verde", "Esto es un libro"). Predicados menos ligados a la percepción son interpretados como ocurren en oraciones que también contienen predicados aprendidos ostensiblemente, o a través de sus relaciones con oraciones que contienen tales predicados.” Davidson, D. (2005a), p. 162. La traducción es mía.

${ }^{12}$ Resulta interesante al respecto el libro de Roberth Myers y Claudine Verheggen (2016), en particular la Parte I.

${ }^{13}$ Véanse Davidson (1989), (1990), (1991a), (2001a), (2001b).

${ }^{14}$ En Davidson (2005b).
} 
un parecido de familia con las desarrolladas por Wittgenstein en su Tractatus. Podríamos decir que su propuesta se dirige a sostener que la necesidad de aceptar ciertas estructuras en nuestro lenguaje nos obliga a aceptar ciertas entidades en nuestra ontología. Aunque en este caso se propone ir más allá de la mera cuestión de decir con qué entidades nos compromete una teoría, en la dirección de qué entidades diríamos que hay. ${ }^{15}$

Su punto de partida es el conocido reconocimiento de que las definiciones de verdad de Tarski, cuando el lenguaje para el cual se está construyendo la definición permite el anidamiento indefinidamente complejo de cuantificadores, lo que presumiblemente, según él, incluye a todos los lenguajes naturales, no hay modo de evitar una semántica de la referencia que relacione sistemáticamente expresiones con objetos. Por cierto que dichas relaciones se dan a través de las variables de cuantificación, con lo cual, al decir de Davidson "para Tarski como para Quine, ser es ser el valor de una variable" (Davidson 1993, p. 41). La cuestión es si un método semántico apropiado puede llevarnos a conclusiones metafísicas respecto de lo que hay. Y su opinión en principio es que sí.

Es cierto que respecto de esta pretensión se presentan dos problemas:

(i) la tesis quineana de la inescrutabilidad de la referencia, que él mismo acepta;

(ii) un problema bastante más serio, a saber, que al describir la semántica, y con ello la ontología, de un lenguaje debemos apelar a entidades que pensamos que existen; pero estas son las entidades que pertenecen a la ontología de nuestro propio lenguaje: "no hay modo de que al hacer semántica podamos progresar más allá de nuestros propios recursos" (Davidson 1993, p. 41); y si este es el caso, por qué pretender que haciendo semántica podemos aprender algo más acerca de lo que existe de lo que podemos aprender simplemente preguntando ¿qué existe?

Su respuesta a la primera pregunta será que admitir la inescrutabilidad de la referencia "hace menos daño a la relación entre semán-

15 "Una cosa es decir que la semántica de un lenguaje nos fuerza a comprometernos con la ontología del lenguaje; ¿pero podemos aprender a partir de esto algo sobre la ontología misma, acerca de lo que hay? Pienso que podemos. Si tenemos la semántica correcta de un lenguaje, los objetos que asignamos a las expresiones del lenguaje deben existir. El método semántico apropiado lleva a las conclusiones metafísicas" (Davidson 1993, p. 40). 
tica y ontología del que podríamos pensar". Y la razón de que sea así es que la inteligibilidad de un lenguaje reside en que lo consideremos capaz de hablar acerca de una gran cantidad de las mismas cosas de las que nosotros hablamos. Como señala: "No podríamos dar sentido a un lenguaje que pudiera hablar acerca de partes no separadas de conejo, pero que no pudiera hablar de conejos". En su opinión, en el peor de los casos, "la inescrutabilidad de la referencia puede llevar a extrañas asignaciones de objetos a las palabras individuales, pero no puede afectar la ontología global con la que encontramos que el lenguaje nos compromete" (Davidson 1993, p. 40). ${ }^{16}$ Estoy básicamente de acuerdo con este diagnóstico de Davidson, y dejo para otra ocasión las razones del mismo. Ahora quiero pasar a considerar la segunda dificultad señalada, la cual sin duda es mucho mayor que la recién considerada.

Como habíamos señalado, el problema aquí es en qué medida la semántica de un lenguaje nos permite sacar consecuencias ontológicas que vayan más allá de señalar la ontología con la cual ese lenguaje nos compromete. Según Davidson, parte de la respuesta reside en el carácter sistemático de la semántica. Si bien es cierto que Davidson reconoce que la convicción de que esto es así resultó finalmente tramposa, no descarta cierta productividad en el intento. ${ }^{17} \mathrm{Y}$ considero que esta productividad apunta a mi sospecha, planteada al principio, de que la radicalidad del no poder ser dicho es mucho mayor en lo que respecta al contenido de las oraciones que en lo que respecta a lo mostrado en la interpretación. Sospecha para la cual creo que podré dar más argumentos un poco más adelante. Pero por el momento deseo detenerme en este segundo problema.

En nuestro lenguaje podemos emitir oraciones como "Las ballenas son mamíferos”, pero la pregunta acerca de con qué entidades nos compromete esta expresión no tiene a primera vista una respuesta obvia. Cuáles son las dificultades con las que me enfrento al hacer esta pregunta, devienen claras cuando pedimos las condiciones de verdad

${ }^{16} \mathrm{Y}$ en la misma línea sostiene: "He insistido durante mucho tiempo que la inescrutabilidad de la referencia, como otras formas de la llamada indeterminación de la traducción, no deben ser vistas como más amenazantes para la objetividad de la interpretación semántica, de lo que la existencia de formas diferentes de medida (Fahrenheit, Centígrado; las pulgadas, centímetros; los nudos, millas por hora) lo es para la objetividad de la medida" (Davidson 1993, p. 40).

17 "Cuando llegué a apreciar por primera vez el poder de la semántica sistemática, me convencí de que debe haber soluciones únicas a muchos de los problemas básicos de la ontología. Esta convicción tramposa se fue desvaneciendo lentamente cuando descubrí que muchos de los principios que había tomado por garantizados que gobernaban la buena práctica semántica podrían ser desafiados. No obstante, sigo convencido de que la disciplina es productiva." (Davidson 1993, p. 41). 
de dicha oración y de otras oraciones que se encuentran relacionadas por patrones de inferencia válidos. La introducción de la cuantificación por parte de Frege, nos permite dar una descripción de la forma lógica de dicho enunciado, a saber, " $\forall x(F x \rightarrow G x)$ ", y de acuerdo con esta forma lógica, si aceptamos que toda la carga referencial del lenguaje recae sobre las variables ligadas, podemos decir que las únicas entidades con las que la oración nos compromete son los objetos. Claro que para que la oración sea verdadera no sólo necesitamos de los objetos sino que necesitamos que todos los objetos de un cierto tipo sean de otro cierto tipo, es decir, necesitamos dar cuenta del papel de los predicados " $\mathrm{x}$ es ballena" y "x es mamífero". Pero, si bien para Frege tales predicados tenían referencia, a saber, funciones cuyos valores eran lo Verdadero o lo Falso, en el caso de Tarski, los mismos no tenían que denotar nada sino tan sólo ser satisfechos por secuencias de objetos. Es decir, la ontología de un lenguaje para quien trabaja con los métodos de Tarski consiste de exactamente las entidades que deben ser los valores de las variables de cuantificación. Este criterio de compromiso ontológico hace que gran parte del trabajo de una semántica sistemática sea el de localizar las posiciones que deben ser ocupadas por las variables. Por cierto que esto sólo nos permite dar la ontología de un lenguaje dado, pero nada nos permite concluir respecto de cuestiones ontológicas generales. Si pudiéramos dar con la única y verdadera forma lógica del lenguaje, entonces podríamos dar ese paso, aunque por cierto estamos muy lejos de poder sostener algo por el estilo. Sin embargo, en opinión de Davidson, es posible excluir algunas propuestas semánticas mostrando que son inconsistentes con exigencias que uno no puede abandonar voluntariamente. Así, por ejemplo, si aceptamos con Davidson que todo lenguaje susceptible de ser aprendido debe tener un vocabulario finito, o que una teoría satisfactoria de la verdad para un lenguaje debe ser finitamente axiomatizable, podemos excluir muchos modos aparentemente atractivos de evitar compromisos ontológicos. Una vez aceptado esto, podríamos aconsejar como método para determinar la ontología el intentar eliminar todas las entidades que podamos: aquellas que se resistan a ser eliminadas son las que conforman la ontología general de todo lenguaje. Claro que esto no sería más que la aplicación del criterio de compromiso ontológico formulado por Quine. Por cierto que es la aplicación de este principio lo que lleva a Davidson a postular eventos como modo de dar cuenta de las cláusulas adverbiales, la forma lógica de las oraciones de acción y la de las oraciones causales. La cuestión es cuál es el modo de dar el salto desde este compromiso que nos impone la aceptación de las oraciones de un lenguaje dado, al 
compromiso que debería imponernos cualquier lenguaje, ya que sólo en este caso podríamos hablar de una ontología a secas. Parece claro que, como sostiene Davidson (1993, p. 42), si una ontología de eventos fuera el único modo de dar un análisis semántico satisfactorio de estas oraciones y de las relaciones entre ellas, tendríamos un argumento muy fuerte a favor de los eventos. Sea o no este el caso, parece que esto muestra de un modo claro cómo las consideraciones semánticas son pertinentes para las preguntas sobre ontología. La cuestión adoptaría la forma siguiente:

(i) el criterio de compromiso ontológico de Quine nos permite determinar con qué tipo de entidades nos compromete un lenguaje dado;

(ii) si ningún lenguaje puede ser interpretable sin apelar a entidades del tipo A, entonces ese tipo de entidades constituye la ontología a secas y no la ontología relativa a un lenguaje dado.

El problema radica naturalmente en cuáles son los criterios para determinar la prótasis de (ii).

Davidson intentó un argumento apoyándose en la idea de que una interpretación semántica correcta de un lenguaje debe hacer a los hablantes de ese lenguaje inteligibles para el intérprete. Este argumento se dirigía a establecer que una interpretación correcta del lenguaje de una persona por otra debe terminar con un amplio grado de acuerdo básico sobre ontología, ambas personas deben estar de acuerdo acerca de qué cosas existen. Si agregamos a esto que algo es un lenguaje si resulta interpretable, se habrá obtenido la ontología que buscamos. Pero el argumento se enfrentó a la objeción de por qué no podría darse el caso de que hablante e intérprete compartieran el error masivo. El intento por parte de Davidson de enfrentar esta objeción fue el apelar a la controvertida hipótesis del intérprete omnisciente. Y, a mi entender, los argumentos en contra de esta hipótesis son lo suficientemente razonables como para no aceptarla. Creo que lo deseable sería dejar de lado el intérprete omnisciente y buscar la salida por otro lado. Considero que un modo en que sería más prometedor buscar una salida sería en los argumentos a favor del carácter mayoritariamente verídico de las creencias, desarrollado en "A Coherence Theory of Truth and Knowledge" de 1983 y otros textos, pero esto sería tema para otro artículo. En lo que sigue prefiero centrarme en cuál debemos suponer que es la naturaleza de la interpretación como para asegurar la clase correcta de acuerdo. A tales efectos, Davidson apela a dos principios metodológicos que constriñen al intérprete a encontrar a un hablante ampliamente correcto. Uno 
que podríamos denominar 'principio de consistencia' y otro que apela a las conexiones causales entre el agente y el medio.

Muy brevemente, el primer principio afirma que la interpretación no puede atribuir al hablante una creencia de la forma "p y no p". Es decir, se debe evitar que la interpretación haga al hablante inconsistente. Respecto de esta afirmación, Moretti podría replicar ${ }^{18}$ que la lógica del hablante podría ser paraconsistente, en cuyo caso al aplicar este principio metodológico correríamos el riesgo de interpretar de modo incorrecto la lógica del lenguaje del hablante. Sin embargo, no creo que este sea el caso, desde el momento en que encuentro que las lógicas paraconsistentes no pueden afirmar de modo razonable "p y no p". Al menos esto sucede en los sistemas paraconsistentes que conozco, como por ejemplo, el sistema $\mathrm{C}_{\mathrm{n}}$ de Da costa, el cual contiene dos negaciones, una débil y otra fuerte y en el cual sólo podría aceptarse "p y no p" a condición de que el 'no' esté por la negación débil, en cuyo caso no estaríamos atribuyendo al hablante la creencia de que "p y no p" puede ser aceptable si 'no' está por la negación fuerte. Por lo tanto, considero que la caracterización de las lógicas paraconsistentes como lógicas inconsistentes pero no triviales no es más que un modo de hablar metafórico y desorientador. ${ }^{19}$

El segundo principio metodológico se apoya en las conexiones causales que se producen entre el agente y el mundo. Esta causalidad tiene para Davidson una dirección doble: del agente al mundo y del mundo al agente. En la acción se ponen de manifiesto los efectos de las creencias del agente sobre el mundo exterior, mientras que en la sensación, como intermediario de la cadena causal que va del mundo a la creencia, lo hacen los constituyentes del mundo. Si bien esto se encuentra trabajado en "A Coherence Theory of Truth and Knowledge" de 1983, y de algún modo en su teoría de la interpretación radical, adquiere una relevancia mayor en lo que hemos denominado el Davidson tardío. Mientras que

${ }^{18}$ Algo que menciona en el artículo citado anteriormente en este trabajo.

${ }^{19}$ Debo admitir que estas afirmaciones no son por todos compartidas y representan un punto de vista propio. Desconozco si hay algún sistema paraconsistente para el cual no se aplique lo que acabo de referir. Sin embargo, no creo que pueda decirse de ningún sistema que no asigne a " $p$ y no $p$ " el valor verdadero, que está entendiendo 'no', o en todo caso ' $y$ ', del modo clásico. Y si este es el caso no tengo derecho a sostener que dichos sistemas permiten afirmar contradicciones. Por otro lado, en caso de que el sistema contenga más de una negación, y una de dichas negaciones sea la negación clásica, no deberíamos considerar dicho sistema como un rival de la lógica clásica sino en todo caso como un sistema complementario o extendido. Eduardo Piacenza me manifestó en una conversación privada que creía que eso era lo que sucedía con el sistema de Da Costa. Creo que incluyó esta observación en algún artículo sobre el tema, pero si es así, no he podido ubicarlo. 
en el Davidson temprano el criterio principal para la corrección de la interpretación es la maximización del acuerdo, en el Davidson tardío, como ya señalamos, se realiza un énfasis especial en la percepción. Y esto se ve en el lugar que la triangulación pasa a tener en la filosofía de Davidson. Mencionada, como señalamos, por primera vez en "Rational Animals" de 1982, recién encontramos un esbozo de argumento en "The Second Person" diez años después. Sin entrar en demasiado detalle, la triangulación, como base para la interpretación, supone la conformación de un triángulo que tiene en uno de sus vértices al hablante, en otro al intérprete y en el tercer vértice, un evento compartido. Esto que llamamos 'evento compartido' es lo que Quine (2000) denomina el 'it', el cual podría ser considerado como el estímulo proximal o el estímulo distal de la cadena que va del ambiente compartido a la creencia. Si adoptamos lo que parece ser la doctrina oficial de Quine, lo compartido son patrones de estimulación; si adoptamos la postura externalista de Davidson, eventos en el mundo. En cualquiera de los dos casos, sin embargo, no hay modo de evitar el estímulo distal. Quine no lo hace cuando trata de explicar la adquisición del lenguaje, aunque sí lo hace cuando de lo que se trata es de dar el significado estimulativo de una expresión. Davidson no lo evita en ningún momento. Incluso en una ocasión Quine llega a afirmar lo siguiente:

Para un infante la oración observacional "Pelota" sería, al principio, simplemente una señal de un estado de cosas recurrente, como "Trueno" o "Hacer frío". Sólo más tarde tiene sentido preguntarse si en la próxima ocasión se trata de la misma pelota o de otra igual a ella. Sólo entonces la reificación ha florecido. Pero la referencia está afuera en el mundo antes y después, en el vértice "ello" del triángulo. (Quine 2000, p. 3, las cursivas y la traducción me pertenecen)

No deja de ser curioso que Quine hable de la referencia "antes" y "después" en el tercer vértice del triángulo. Un modo de entenderlo sería considerando que la referencia "antes" alude al estímulo distal y "después" a la reificación a partir del estímulo proximal, vía adquisición del aparato de individuación. Sin embargo, esto, pese a configurar una diferencia significativa con respecto a Davidson, podría parecer no del todo decisivo si pensamos que él admite la tesis quineana de la inescrutabilidad de la referencia. De todas formas, como señalé anteriormente, el alcance de la inescrutabilidad es mucho menor en Davidson que en Quine. Como vimos en su momento, en opinión de Davidson, la inescrutabilidad de la referencia puede llevar a lo sumo 
a extrañas asignaciones de objetos a las palabras individuales, pero no puede afectar la ontología global con la que encontramos que el lenguaje nos compromete. Esto es similar a decir, parafraseando a Putnam pero diciendo lo contrario de lo que él dice, que el lector de Word and Object debe salir con la idea correcta de que toda interpretación alternativa está de algún modo vinculada con la interpretación estándar. Y la razón por la cual Putnam puede llevar adelante su argumento en contra de esta conclusión es, como he señalado en otra parte ${ }^{20}$, que deja de lado precisamente la situación de triangulación en la cual la percepción juega un papel tan fundamental. Veamos cuál es ese papel.

$\mathrm{Al}$ hablar de triangulación debemos establecer una diferencia entre dos tipos de triangulación considerados por Davidson. ${ }^{21} \mathrm{Un}$ tipo de triangulación que se da entre animales que carecen de lenguaje, es decir, una triangulación pre-conceptual, a la que Davidson llama 'primitiva', y una triangulación que es la fuente del aprendizaje del lenguaje y de la interpretación, una triangulación plenamente lingüística. En "Externalism" Davidson da el siguiente ejemplo de triangulación primitiva:

Observo una leona acechando a una gacela. Describo esta situación del siguiente modo correlacionando dos aspectos de la situación: la conducta de la gacela y la conducta de la leona (la leona cambia de curso para interceptar a la gacela; la gacela cambia de curso para evadir a la leona). Confirmo mi teoría de que la gacela es el objeto saliente para la leona (ente otras posibilidades) observando la respuesta de otras leonas a gacelas en otras ocasiones. Pero incluso esta evidencia adicional deja en mis manos decidir cuál es el objeto de atención de la leona; yo decido sobre la base de lo que captura mi atención. Si una segunda leona se une a la primera en persecución de la gacela, puedo eliminar la dependencia completa del objeto saliente de mi propia elección de la manera siguiente: clasifico juntas las respuestas de la leona A con las respuestas de la leona B en el mismo lugar y en el mismo tiempo. El foco de las causas compartidas es ahora lo que tomo como siendo el objeto saliente para ambas leonas. Ya no tengo que depender de mi propia elección del estímulo relevante de la conducta de las leonas. En el caso excepcional en el que las respuestas difieren puedo decir: una de las leonas se ha

\footnotetext{
${ }^{20}$ Véase Caorsi (2013).

${ }^{21}$ Aunque es necesario reconocer que no siempre hace esta distinción con la claridad suficiente.
} 
equivocado. El desafío es colocar las leonas en posición de distinguir estos casos. Para hacerlo tenemos que eliminar la dependencia de mi elección arbitraria (o interesada) de las respuestas relevantemente similares de parte de las leonas. Se introduce un elemento adicional cuando las leonas cooperan para arrinconar a su presa. Cada una mira a la otra mientras ambas miran a la gacela, observando las reacciones de la otra a los cambios de dirección.” (Davidson 2001, pp. 6-7, la traducción me pertenece)

$\mathrm{Al}$ describir este ejemplo es necesario ubicarse en dos situaciones, una primera en la cual el observador no participante es el que determina cuál es el objeto saliente al cual la leona responde, y un segundo caso en que dicha determinación ya no cae bajo la órbita del observador no participante, sino que queda determinada por el accionar de los individuos que participan en la situación: en este caso, las dos leonas. La distinción entre ambos casos me parece de importancia fundamental, en la medida en que permite describir una triangulación entre animales no hablantes que son capaces de determinar el objeto compartido de su percepción y actuar en consecuencia. Aquí, ambas leonas no sólo se enfocan en la gacela, sino que se perciben una a la otra y actúan como consecuencia de dichas percepciones. En lo que nosotros describiríamos como el intento de capturar la gacela, las leonas desarrollan una triangulación que determina su acción, uno de cuyos vértices es la gacela y los otros dos, cada una de las leonas. En este caso tenemos una situación que no depende del lenguaje ni de la descripción que podamos hacer de ella. La situación básica, como ha quedado expuesta, contiene un mínimo de tres elementos: dos criaturas y un mundo de objetos, propiedades y eventos que las criaturas pueden discriminar en la percepción. Y, como remarca Davidson, "esta causa común (la gacela en este ejemplo) sería típicamente algún rasgo objetivo del entorno. Nada en esta historia sugiere la necesidad de pensamiento, conceptualización, o error, aunque la criatura falle ocasionalmente en actuar en concierto" (Davidson 2001, p. 6). Como vemos por este ejemplo y las consideraciones posteriores, este tipo de triangulación primitiva ni se refiere al significado ni depende del lenguaje para darse. Es algo que se muestra independientemente del lenguaje. Pero esto que se muestra, es, en opinión de Davidson, una condición necesaria para la adquisición de la conceptualización, el pensamiento y el habla. ${ }^{22}$

${ }^{22}$ Dice Davidson: "He aquí mi tesis: un triángulo interconectado como este (dos leonas, una gacela) constituye una condición necesaria para la existencia de la 
Para que se den el pensamiento y el habla es necesario que la triangulación se haga más compleja. Primero, debemos incluir como dos de los vértices a individuos capaces de desarrollar un pensamiento conceptual, que tengan el concepto de objetividad y que puedan determinar los aspectos de la causa que son determinantes para fijar el significado, entre otras cosas. El argumento de la triangulación tiene como finalidad dar cuenta de cómo esto es posible. Sin embargo, como no es el objeto de estas páginas realizar un análisis de este argumento, y considero que ya me he explayado más de lo necesario sobre el concepto de triangulación, me contento con las cuestiones ya mencionadas. El objetivo de este breve desarrollo está vinculado con lo que el acto de enunciación muestra, es decir, la estructura intérpretescosas-relaciones, dado que considero que el proceso de la triangulación descrito por Davidson es un modo de describir dicha estructura. O sea, es una forma de decir acerca de dicha estructura.

Quedaría por considerar si dicho decir cae en algunos de los dos ítems de la dicotomía:

(i) parte de lo que puede decirse;

(ii) lo que posibilita todo decir.

Y considero que cae en ambos. Por un lado, enuncia situaciones que, sin involucrar elementos semánticos más allá de los que obviamente usamos para describirlas, son presupuestas por el habla. En una ocasión Austin señaló que para que el lenguaje fuera posible el mundo debería exhibir similitudes y diferencias; lo que hemos estado diciendo acerca de la triangulación presupone estas y agrega varias más. El caso de la triangulación primitiva es un caso de una teoría $\mathrm{M}$, del tipo de la mencionada por Moretti, pero, como lo señalé en su momento, no veo en ella ninguna de las dificultades que él menciona. Y tal vez podría decirse lo mismo de la triangulación semántica, aunque en este caso no me asiste la misma seguridad.

Por otro lado, creo que estas descripciones constituyen un ejemplo de la gradación, que señalé más arriba, respecto de poder decir lo que se muestra. En este caso hay algo que la enunciación muestra sobre lo cual se puede hablar. Y creo que la triangulación primitiva es una condición necesaria, aunque no suficiente, para la existencia del lenguaje, la cual puede ser enunciada aunque el lenguaje la presuponga. Considero que la triangulación primitiva muestra la capacidad de distinguir rasgos objetivos del entorno, que resultan necesarios para que el lenguaje sea posible, sin necesidad de apelar al pensamiento,

conceptualización, el pensamiento y el lenguaje" (2001b, p. 7).

ANÁLISIS FILOSÓFICO 40 Nº ESPECIAL (diciembre 2020) 
la conceptualización o el significado. También creo que la posición de Davidson se puede incluir dentro de las dos perspectivas mencionadas por Moretti, IL y ML, aunque por momentos parece que él sostendría lo mismo. Si estoy en lo cierto, entonces, dentro de las cosas que se muestran, unas se pueden decir y otras no. Si no estoy en lo cierto, tal vez nos quedaría el consuelo de decir con Wittgenstein (1921, 6.54): "[Estas] proposiciones esclarecen porque quien me entienda las reconoce al final como absurdas. Cuando a través de ellas -sobre ellas- ha salido fuera de ellas. (Tiene, por así decirlo, que arrojar la escalera después de haber subido por ella.)"

\section{Bibliografía}

Caorsi, C. (2013). Dos modelos de interpretación: la indeterminación de la traducción de Quine y el argumento modelo-teorético de Putnam. Manuscrito, 36(1), 49-65.

Davidson, D. (1989). The conditions of thought. En Le Cahier du Collège International de Philosophie (pp. 165-71). Editions Osiris.

Davidson, D. (1990). Meaning, truth and evidence. En R. Barrett \& R. Gibson (Eds.), Perspectives on Quine (pp. 68-79). Blackwell.

Davidson D. (1991a). Epistemology externalized. Dialectica, 445, 191202. (Originalmente en español, 1990.)

Davidson, D. (1991b). Three varieties of knowledge. En A. Phillips Griffiths (Ed.), A. J. Ayer: memorial essays, Royal Institute Philosophy Supplement 30 (pp. 153-66). Cambridge University Press.

Davidson, D. (1993). Method and metaphysics. En Truth, language, and history. Clarendon Press.

Davidson, D. (2001a). The second person. Midwest Studies in Philosophy, 17, 255-67.

Davidson, D. (2001b). Externalism. En P. Kotatko, P. Pagin, \& G. Segal (Eds.), Interpreting Davidson (pp. 1-16). CLSI.

Davidson, D. (2005a). Truth and predication. Cambridge, The Belknap Press of Harvard University Press.

Davidson, D. (2005b). Truth, language and history. Clarendon Press.

Føllesdall, D. (1999). Triangulation. En L.W. Hahn (Ed.), The Philosophy of Donald Davidson. The Library of Living Philosophers, vol. XXVII (pp. 719-732). Open Court.

Kotatko, P., Pagin, P. \& Segal, G. (2001). Interpreting Davidson. CLSI.

Moretti, A. (2014). La unidad proposicional. Avatares filosóficos, 1, 62-72. https://www.researchgate.net/publication/311714568 
Myers, Roberth \& Verheggen, Claudine. (2016). Donald Davidson's triangulation argument, A Philosophical Inquiry. Routledge.

Russell, B. (1910). Conocimiento directo y conocimiento por descripción. En Misticismo y lógica (pp. 285-314). Edhasa.

Quine, W. (1960). Word and object. Cambridge University Press.

Quine, W. (2000). I, you and it: an epistemological triangle. En A. Orenstein \& P. Kotatko (Eds.), Knowledge, language and logic: questions for Quine (pp. 1-6). Kluwer Academic Publishers.

Wittgenstein, L. (1921). Tractatus logico-philosophicus. Traducción española de Jacobo Muñoz e Isidoro Reguera. Alianza. 